\title{
Measurement of non-purified GPCR thermostability using the homogenous ThermoBRET assay
}

Bradley L. Hoare ${ }^{1,2}$, Amandeep Kaur ${ }^{1,2}$, Clare R. Harwood ${ }^{1,2}$, Nicola C. Dijon ${ }^{1,2}$, Nicholas D. Holliday ${ }^{1,2}$, David A. Sykes ${ }^{1,2,{ }^{*}}$ and Dmitry B. Veprintsev ${ }^{1,2, *}$

${ }^{1}$ Centre of Membrane Proteins and Receptors (COMPARE), University of Birmingham and University of Nottingham, Midlands, UK

2Division of Physiology, Pharmacology \& Neuroscience, School of Life Sciences, University of Nottingham, Nottingham, NG7 2UH, UK

"correspondence should be addressed to david.sykes@nottingham.ac.uk and dmitry.veprintsev@nottingham.ac.uk

\section{ABSTRACT}

Sensitive assays to measure the thermostability of membrane proteins are important tools in protein purification optimisation and drug discovery. Here, we present a ThermoBRET method to quantify the relative thermostability of $G$ protein coupled receptors (GPCRs), using cannabinoid receptor $2\left(\mathrm{CB}_{2}\right)$ as an example. This method applies the principles of Bioluminescence Resonance Energy Transfer (BRET) between Nanoluciferase (Nluc) and a thiol-reactive fluorescent dye that covalently binds cysteines in the GPCR transmembrane domain, exposed by unfolding. We demonstrate that the melting point $\left(\mathrm{T}_{\mathrm{m}}\right)$ of Nluc-fused GPCRs can be determined in non-purified detergent solubilised membrane preparations, revealing differences in thermostability for different detergent solubilising conditions and in the presence of stabilising ligands. In addition, we extended the range of the assay by developing the thermostable tsNLuc by incorporating mutations from the fragments of split-Nluc $\left(T_{m}\right.$ of $87^{\circ} \mathrm{C}$ vs $59^{\circ} \mathrm{C}$ ). ThermoBRET allows the high-throughput determination of GPCR thermostability which will be useful for protein purification optimisation strategies and as part of a drug discovery screening platform. 


\section{Introduction}

G protein coupled receptors (GPCRs) are a large family of membrane proteins that are important drug discovery targets (Hauser, Attwood et al. 2017). Structural and biophysical studies of GPCRs have significant importance in modern drug discovery (Congreve, de Graaf et al. 2020) but require receptor solubilisation from their native membrane environment and subsequent purification. Optimisation of receptor stability during this process is a key component to success (Tate 2010). Additionally, the ability of a bound ligand to stabilise the receptor structure is a property which can be exploited in screening efforts to find novel drug candidates (Fang 2012, Zhang, Stevens et al. 2015). Low abundance of GPCRs even in over-expressing systems and their inherently low stability in detergents (Milic and Veprintsev 2015) requires sensitive protein stability assays that can be used without protein purification for progress in structural biology and drug discovery efforts.

Recently, we reported a ThermoFRET assay that uses the principles of time-resolved Förster resonance energy transfer (TR-FRET) between a Tb3+ labelled receptor and a thiol-reactive fluorescent dye that covalently binds to the cysteines exposed upon temperature induced unfolding of a GPCR (Tippett, Hoare et al. 2020). The extreme distance dependence of FRET (usually occurring only where donor:acceptor distances are $<10 \mathrm{~nm}$ ) allows the detection of bimolecular proximity in a homogenous manner, omitting purification steps to remove components which confound traditional measurements.

Here, we present the ThermoBRET assay based on bioluminescence resonance energy transfer between the bright Nanoluciferase (Nluc, and, correspondingly, NanoBRET) (Hall, Unch et al. 2012), acting as a donor of light and a thiol reactive Sulfo-Cyanine3 maleimide (SCM), the acceptor, allowing us to quantify the relative thermostability of non-purified GPCRs solubilised into detergent micelles. As a test case we focus on cannabinoid receptor $2\left(\mathrm{CB}_{2} \mathrm{R}\right)$ as a therapeutically promising (Pertwee 2012) but unstable drug target (Vukoti, Kimura et al. 2012, Beckner, Gawrisch et al. 2019, Beckner, Zoubak et al. 2020). This assay is significantly more sensitive and is easier to use compared to existing methodologies. 


\section{Results}

\section{ThermoBRET provides reliable measurements of GPCR stability}

We fused a version of donor Nluc to the receptor N-terminus, preceded by a signalling peptide to ensure its successful expression and Twin-strep affinity tag for a possibility of protein purification (Supplementary Information 4). Detergent solubilised receptor samples containing a thiol reactive Sulfo-Cyanine3 maleimide (SCM) acceptor are incubated at varying temperatures using a gradient forming PCR thermocycler. As the receptor unfolds on heating the SCM covalently binds to exposed cysteine residues (Figure 1). We chose SCM because of its suitability as a BRET acceptor for Nluc, water solubility, and relatively low cost compared to other thiol-reactive fluorophores. In principle, any maleimide or other thiol-reactive conjugated fluorescent dye with overlapping donor-acceptor emission-absorption spectra can be used. The unfolded state of the receptor due to thermal denaturation is measured as NanoBRET between the Nluc tag and the SCM acceptor, and is quantified as a ratio of the donor and acceptor light emissions, termed the NanoBRET ratio. The relative thermostability of a receptor in different solubilised non-purified membrane preparations can be easily determined, first by thermal denaturation across a temperature gradient on a thermocycler block, rapid cooling to $4{ }^{\circ} \mathrm{C}$, and then following the addition of the Nluc substrate furimazine and measurement of the NanoBRET ratio in a 384-well luminescence plate reader at room temperature (Figure 1). The midpoint of the transition curve is found by fitting the data to a Boltzmann sigmoidal equation to obtain a $T_{m}$.

When solubilised in DDM detergent, $\mathrm{CB}_{2}$ had a $\mathrm{T}_{\mathrm{m}}$ of around $33^{\circ} \mathrm{C}$ (Figure $2 \mathrm{~A}$ ) and was marginally more stable in LMNG $\left(\mathrm{T}_{\mathrm{m}}=35^{\circ} \mathrm{C}\right)$. Addition of CHAPSO and the cholesterol derivative $\mathrm{CHS}$ in the detergent micelles provided the highest thermostability for $\mathrm{CB}_{2}\left(\mathrm{~T}_{\mathrm{m}}=43{ }^{\circ} \mathrm{C}\right.$ in $\left.\mathrm{LMNG} / \mathrm{CHAPSO} / \mathrm{CHS}\right)$. This observation is consistent with the reported increase in $\mathrm{CB}_{2}$ stability in DDM/CHAPSO mixed micelles(van Veen, Vukoti et al. 2012). Differences in the detergent stability of the adrenergic $\beta_{2}$-receptor were also found (Supplementary Figure 1). Hence, this assay can be readily used to screen for the best detergent solubilising conditions before attempting a large-scale purification.

We also tested a selection of endogenous and synthetic cannabinoid receptor ligands for their ability to increase the thermostability of $\mathrm{CB}_{2}$ (Figure $2 \mathrm{~B}$ ). The lipophilicity of its ligands has made the $\mathrm{CB}_{2}$ receptor a particularly challenging target for ligand binding experiments due to their high non-specific binding. These ligands were all tested at a concentration of $20 \mu \mathrm{M}$, well above their dissociation constant $\left(K_{\mathrm{D}}\right)$ at room temperature, in order to ensure full occupancy of the solubilised receptors. Interestingly, the endogenous cannabinoid 2-arachidonoylglycerol (2AG) increased the $\mathrm{T}_{\mathrm{m}}$ of $\mathrm{CB}_{2}$ by around $6{ }^{\circ} \mathrm{C}$, whereas the other endogenous cannabinoid anandamide (AEA) only increased the $T_{m}$ by around $2{ }^{\circ} \mathrm{C}$. The most probable reason for these observations are the variable temperature dependence of the affinity of the ligand to the receptor as well as well as the degree of the entropy contribution to the binding (Layton and Hellinga 2010). Other synthetic cannabinoid ligands HU308 and SR144528 also produced appreciable increases in thermostability, and the pattern of 
ligand stabilisation appeared different for the related $\mathrm{CB}_{1}$ receptor (Supplementary Figure 2).

\section{tsNluc extends the range of the ThermoBRET assay}

One problematic aspect of the ThermoBRET is the thermostability of the Nluc donor itself, which has been reported to unfold at around $55-60^{\circ} \mathrm{C}$. This limits the thermal range for this assay and prevents accurate $T_{m}$ determination in conditions where the receptor itself is particularly thermostable, for example when $\mathrm{CB}_{2}$ is bound to the high affinity non-selective cannabinoid agonist HU210 (Figure 2C). We therefore combined Nanoluc mutations which had been developed by Promega as part of their efforts to create a stable split-luciferase system (Dixon, Schwinn et al. 2016) and found that these mutations improved thermostability of the full length luciferase by about $30{ }^{\circ} \mathrm{C}$ (Figure 2D). In line with previous reports (Hall, Unch et al. 2012) we found that purified Nanoluc had a $T_{m}$ of $59^{\circ} \mathrm{C}$, and that purified thermostable Nluc (tsNluc) had a $T_{m}$ of $87^{\circ} \mathrm{C}$ (Figure 2D), making it preferable for thermostability measurements across a wide temperature range. Importantly, tsNluc contains no cysteine residues (Supplementary Information 3) and thus is unaffected by maleimide/thiol chemistry. Applying this novel tsNluc fusion improved the working temperature range of the ThermoBRET assay and allowed successful $T_{m}$ determination for $\mathrm{CB}_{2}$ in the presence of HU210 (Figure 2E). Strikingly, HU210 was able to stabilise $\mathrm{CB}_{2}$ by around $12{ }^{\circ} \mathrm{C}$, the highest level achieved of any of the ligands tested.

\section{Discussion}

The processes of protein unfolding and protein aggregation are related, yet very separate, phenomena. Nluc has already been successfully used in similar applications which monitor protein aggregation. Such applications fuse either full length Nluc (Dart, Machleidt et al. 2018) or a split Nluc (Martinez, Asawa et al. 2018) fusions to the protein of interest and monitor the decrease in luminescence activity to measure aggregation of the protein of interest after thermal denaturation. In contrast, the ThermoBRET assay described here captures the initial conformational unfolding events which expose maleimide reactive cysteine residues in the protein of interest. In addition, ThermoBRET measurement is buffered from changes in the concentration of the luciferase fused target protein because a ratiometric method is used to calculate resonance energy transfer, contrasting with assays which measure luminescence intensity only. This means the measurement should remain robust even at low concentrations of the target protein, or when technical variability becomes problematic.

We note that under the test conditions we used, the luminescence activity of just 85 pM purified Nluc and tsNluc was easily measured in a 96-well plate (Figure 2D), making the assay extremely sensitive. This makes the assay particularly amenable to high-throughput screening attempts where there are limitations on the amounts of reagent that can be provided, for example protein targets which are poorly expressed in vitro and/or in vivo. This assay principle could even be applied in more physiologically relevant in vivo cellular models whereby tsNluc (or the 11 amino acid 
HiBiT tag) is fused to an endogenously expressed protein via CRISPR-mediated insertion (White, Johnstone et al. 2019).

The novel thermostable tsNluc we describe has clear advantages compared to Nluc. Firstly, due to its improved thermostability it is less likely to unfold before the protein of interest and cause sample aggregation and other possible artefacts. Secondly, whilst previous reports (Hall, Unch et al. 2012) and our own data (Figure 2D) showed the $\mathrm{T}_{\mathrm{m}}$ of Nluc to be $59^{\circ} \mathrm{C}$, more recent use of Nluc to monitor protein aggregation show clear luminescence activity after protein samples had been heated to temperatures $>60{ }^{\circ} \mathrm{C}$ before cooling (Dart, Machleidt et al. 2018). We speculate that Nluc has propensity to spontaneously refold after thermal denaturation, and that the presence of detergents in the buffers of the latter report either aided Nluc refolding or delayed irreversible protein aggregation. In our ThermoBRET assays, the cysteine exposed upon Nluc unfolding would potentially react with the SCM and prevent its refolding, whereas tsNluc avoids these pitfalls. The cysteine-less sequence of tsNluc also allows easy in vitro chemical tagging of tsNluc-fusions. By introducing a new cysteine within the sequence, any molecule could be conjugated to tsNluc by thiolreactive chemistry, enhancing its potential for protein engineering and enabling a wide scope for future applications.

In comparison to our previous ThermoFRET application using a Tb3+ labelled receptor as a FRET donor (Tippett, Hoare et al. 2020), the ThermoBRET approach offers potential advantages. ThermoFRET requires cell surface labelling of the receptorfused SNAP tag with the Tb3+ donor molecule, adding to assay cost but perhaps more importantly creating an extra labelling step that can be problematic if the tag is not readily exposed. In contrast, the use of a genetically encoded bioluminescent donor (ie. tsNluc or Nluc) omits this labelling step. This means that fused proteins which are poorly trafficked and their SNAP-tag are not exposed are now amenable as they do not require labelling at the cell surface. Additionally, TR-FRET requires more sophisticated detection by plate readers with time-resolved fluorescence detection capabilities, whereas BRET only requires a luminometer with filtered light detection that are readily available in many labs.

The ability of the ThermoBRET assay to quantify ligand-induced changes in the $T_{m}$ of the receptor makes it an ideal tool to study ligand binding to GPCRs. In principle, this assay can detect compounds which bind the target at any site, assuming this interaction influences the thermodynamic conformational landscape of the protein. It can be used to screen potential ligands for orphan GPCRs as it does not depend on the availability of tool compounds or known binders to develop a competition assay. Moreover, it could be used to detect the combined stabilisation of several ligands to discover positive and negative allosteric modulators of GPCRs.

Additionally, the high thermostability and cysteine-less sequence of tsNluc makes it a widely applicable tool for biochemical fusion to any molecule of interest via well described cysteine conjugation strategies. 
Overall, ThermoBRET is an excellent and highly sensitive tool for optimisation of solubilisation conditions and for higher throughput biophysical screening of GPCR compound libraries to support structural biology and drug discovery efforts.

\section{METHODS}

\section{Drug compounds and reagents}

Sulfo-Cy3 maleimide (SCM) (Lumiprobe GmbH, Germany) was obtained in powder form, dissolved in DMSO at a concentration of $10 \mathrm{mM}$ and stored in the dark at -20C. Furimazine, the substrate for Nluc, was obtained from the Nano-Glo Luciferase Assay System kit (Promega, UK) provided at a concentration of $5 \mathrm{mM}$. Cannabinoid ligands (anandamide [AEA], 2-arachydonyl-glycerol [2AG], SR144528, HU210, HU308, cannabinol) were obtained from Tocris Bioscience and dissolved in DMSO to a storage concentration of $10 \mathrm{mM}$, except $A E A$ and $2 A G$ which were dissolved in $\mathrm{EtOH}$. Rimonabant was obtained from Roche Pharmaceuticals GmbH (Germany).

\section{Plasmid construction}

For mammalian cell expression, receptor constructs were cloned into pcDNA4/TO using Gibson assembly (Gibson, Young et al. 2009). All GPCR constructs contained an N-terminal cleaved signal peptide to improve expression, followed by a Twin-Strep affinity tag, then Nluc (or tsNluc) followed by the receptor sequence. The synthesized cDNA for tsNluc was obtained from GeneArt Gene synthesis (Invitrogen). For bacterial cell expression of Nluc and tsNluc, cDNA sequences were cloned into the pJ411 expression plasmid with a N-terminal 10X histidine affinity tag and TEV cleavage site encoded before the protein of interest. Amino acid sequences of the constructs used are provided in Supplementary Information 4. The correct sequence within the expression cassette of all plasmid constructs was verified by Sangar sequencing (Genewiz, UK).

\section{Mammalian cell culture}

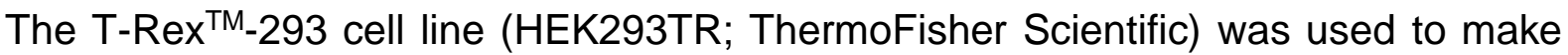
stable expressing cell lines for receptors cloned into pcDNA4/TO. HEK293TR cells were cultured in growth medium (DMEM, 10\% FCS, $5 \mu \mathrm{g} / \mathrm{mL}$ blasticidin) in a $37^{\circ} \mathrm{C}$ humidified incubator with $5 \% \mathrm{CO}_{2}$. Stable cell lines were generated by PEI transfection of pcDNA4/TO plasmids into HEK293TR cells. 24-48 hours after transfection, 20 $\mu \mathrm{g} / \mathrm{mL}$ zeocin was incorporated into the growth medium until stable expressing, zeocin-resistant cell populations remained (2-4 weeks). To produce cells for membrane preparations, 1 X T175 culture flask of confluent stable cells were treated with $1 \mu \mathrm{g} / \mathrm{mL}$ tetracycline for $48 \mathrm{~h}$ to induce receptor expression. Following this, cells were lifted by trituration and centrifuged at $500 \mathrm{G}$ for 10 minutes. Cell pellets were then frozen at $-80{ }^{\circ} \mathrm{C}$ until membranes were prepared.

\section{Membrane preparations}

HEK293TR cell pellets were resuspended in $20 \mathrm{~mL}$ of ice cold buffer (10 mM HEPES $\mathrm{pH}$ 7.4, $10 \mathrm{mM}$ EDTA) and homogenised using a Ultra Turrax (Ika Work GmbH, Germany). The homogenised cell suspension was then centrifuged at $4{ }^{\circ} \mathrm{C}$ for 5 
minutes at $500 \mathrm{G}$ to remove whole cells and large debris, and the remaining supernatant was then centrifuged twice at $4{ }^{\circ} \mathrm{C}$ and $48,000 \mathrm{G}$ for 30 minutes before the membrane pellet was resuspended in buffer (10 mM HEPES pH 7.4, $0.1 \mathrm{mM}$ EDTA). Protein concentration of resuspended membranes was determined with using a Pierce BCA Protein assay kit (ThermoFisher Scientific) and was adjusted to $3-10$ $\mathrm{mg} / \mathrm{mL}$ before being aliquoted and stored at $-80^{\circ} \mathrm{C}$.

\section{ThermoBRET Thermostability experiments}

The CORE buffer for thermostability experiments contained $20 \mathrm{mM}$ HEPES pH 7.5, $150 \mathrm{mM} \mathrm{NaCl}, 10 \% \mathrm{w} / \mathrm{v}$ glycerol, $0.5 \% \mathrm{w} / \mathrm{v}$ BSA. Cell membranes were diluted in CORE buffer to approximately $0.1-0.5 \mathrm{mg} / \mathrm{mL}$ total protein, and were then centrifuged at $16,000 \mathrm{G}$ for 60 minutes at $4{ }^{\circ} \mathrm{C}$ to remove residual EDTA from the membrane preparation buffers. Membrane pellets were then resuspended in CORE buffer containing detergent, and samples were incubated at $4{ }^{\circ} \mathrm{C}$ with gentle shaking for $1 \mathrm{~h}$ to solubilise membranes. Detergent/cholesterol concentrations used were either $1 \%$ DDM, $1 \%$ DDM / $0.5 \%$ CHAPSO / 0.3\% CHS, 0.5\% LMNG, or 0.5\% LMNG / $0.5 \%$ CHAPSO, $0.3 \%$ CHS. Samples were then centrifuged again at $16,000 \mathrm{G}$ for 60 minutes at $4{ }^{\circ} \mathrm{C}$ to remove unsolubilised material, and the resulting supernatant containing detergent micelles was transferred to a fresh tube. These supernatants were then kept on ice for up to 48 hours during testing. For thermostability testing, solubilised receptors were diluted 10-fold in CORE buffer with the addition of $1 \mu \mathrm{M}$ SCM and $20 \mu \mathrm{M}$ of ligand (if used). This was incubated on ice for 15 minutes before being aliquoted across 96 -well PCR plates and placed in the pre-cooled $\left(4{ }^{\circ} \mathrm{C}\right)$ PCRmax Alpha Cycler 2 Thermal Cycler (Cole-Palmer Ltd, St. Neots, UK). Samples were then incubated at different temperatures for 30 minutes via a temperature gradient across the plate. Following rapid cooling of the samples to $4^{\circ} \mathrm{C}$, samples were then transferred to white 384 well proxiplates (Perkin Elmer) containing furimazine at a final concentration of $10 \mu \mathrm{M}$. The plate was then read using a PHERAstar FSX plate reader (BMG) at room temperature using the 450BP80/550LP filter module. Measurements were performed in singlet for each temperature point.

\section{Nanoluc and tsNluc expression and purification}

NiCo21(DE3) chemically competent E. coli were transformed with pJ411 bacterial expression plasmids and plated onto LB/agar plates containing $2 \% \mathrm{w} / \mathrm{v}$ glucose and $50 \mu \mathrm{g} / \mathrm{mL}$ kanamycin. After incubation at $37^{\circ} \mathrm{C}$ for $16-24$ hours, a single colony was picked to inoculate $20 \mathrm{~mL}$ of terrific broth containing $0.2 \% \mathrm{w} / \mathrm{v}$ glucose and $50 \mu \mathrm{g} / \mathrm{mL}$ kanamycin. After 16-24 hours in a shaking incubator set at $37^{\circ} \mathrm{C}, 15 \mathrm{~mL}$ of overnight culture was added to $3 \mathrm{~L}$ of terrific broth containing $0.2 \% \mathrm{w} / \mathrm{v}$ glucose and $50 \mu \mathrm{g} / \mathrm{mL}$ kanamycin. This culture grown in a shaking incubator at $37^{\circ} \mathrm{C}$ until an $\mathrm{OD}_{600}$ of $0.7-$ 1 was obtained, and $500 \mu \mathrm{M}$ of isopropyl- $\beta$-D-thiogalactopyranoside (IPTG; VWR Chemicals) was added to induce protein expression. Cells were then grown overnight $\left(16-20\right.$ hours) at $25^{\circ} \mathrm{C}$ in a shaking incubator before being harvested by centrifugation and frozen at $-80{ }^{\circ} \mathrm{C}$. Cell pellets were then thawed on ice, and resuspended in $100 \mathrm{~mL}$ lysis buffer $(100 \mathrm{mM}$ Tris pH 7.5, $300 \mathrm{mM} \mathrm{NaCl}, 0.25 \mathrm{mg} / \mathrm{mL}$ chicken lysozyme, $1 \mu \mathrm{g} / \mathrm{mL}$ bovine DNAse I, $4 \mathrm{mM} \mathrm{MgCl}$, and 3 cOmplete $^{\mathrm{TM}} \operatorname{mini}$ EDTA-free Protease Inhibitor cocktail tablets (Roche)). After $1 \mathrm{~h}$ on ice in lysis buffer, cells were then lysed further by French press. Cell lysates were then clarified by 
centrifugation at 25,000 rcf for 30 minutes and then by passing through a $0.45 \mathrm{uM}$ syringe filter. The His-tagged proteins from the resulting lysate were then purified using a $5 \mathrm{~mL}$ HiTrap TALON Crude column on an ÄKTA start protein purification system (Cytiva Life Sciences) and eluted with $150 \mathrm{mM}$ imidazole. Elution fractions were analysed by SDS-PAGE and fractions which contained no visible contaminants proteins were pooled together. Protein concentration was determined by $A_{280}$ measurement on a Denovix DS-11 FX series spectrophotometer assuming the calculated molar extinction coefficient $\left(\varepsilon_{280}\right)$ of $26,930 \mathrm{~mol}^{-1} . \mathrm{cm}^{-1}$ for both proteins.

\section{Luminescence activity thermostability experiments}

Purified Nluc and tsNluc proteins were serial diluted in 10-fold dilutions from around $200 \mu \mathrm{M}$ down to $100 \mathrm{pM}$ in CORE buffer. Proteins were then aliquoted across 96-well PCR plates (100 uL per well) and placed in the pre-cooled $\left(4^{\circ} \mathrm{C}\right)$ PCRmax Alpha Cycler 2 Thermal Cycler (Cole-Palmer Ltd, St. Neots, UK). Samples were then incubated at different temperatures for 30 minutes via a temperature gradient across the plate. Following rapid cooling to $4{ }^{\circ} \mathrm{C}, 85 \mathrm{uL}$ of samples were then transferred to white 96 well plates (Perkin Elmer) containing $15 \mathrm{uL}$ of diluted furimazine to a final concentration of $10 \mu \mathrm{M}$. After 30 seconds of gentle shaking, the luminescence intensity was measured in a PHERAstar FSX plate reader at room temperature. Measurements were performed in triplicate for each temperature point, and three independent experiments were performed.

\section{Curve fitting and data analysis}

All curve fitting and data manipulation was performed using GraphPad Prism 8. For ThermoBRET measurements, NanoBRET ratio was defined as the 550LP emission divided by the 450BP80 emission. In situations in which the NanoBRET ratio decreased at high temperatures (presumably due to protein aggregation and loss of signal), the data was manually truncated after the highest point. Data was then normalised to the upper and lower datapoints and fitted using a Boltzmann sigmoidal equation constrained to upper and lower values of $0 \%$ and $100 \%$. For luminescence thermostability measurements, unfiltered luminescence was normalised to the top point of the dataset and fitted using a Boltzmann sigmoidal equation with no constraints.

\section{Acknowledgements}

AK was funded by a COMPARE team science summer studentship (2019) awarded to $\mathrm{BH}$. This research was supported by COMPARE funding to DBV. We would like to thank Uwe Grether (Roche Pharma Research \& Early Development, Roche Innovation Center Basel, F. Hoffmann-La Roche Ltd., 4070 Basel, Switzerland) for supplying the $\mathrm{CB}_{1}$ antagonist rimonabant. 

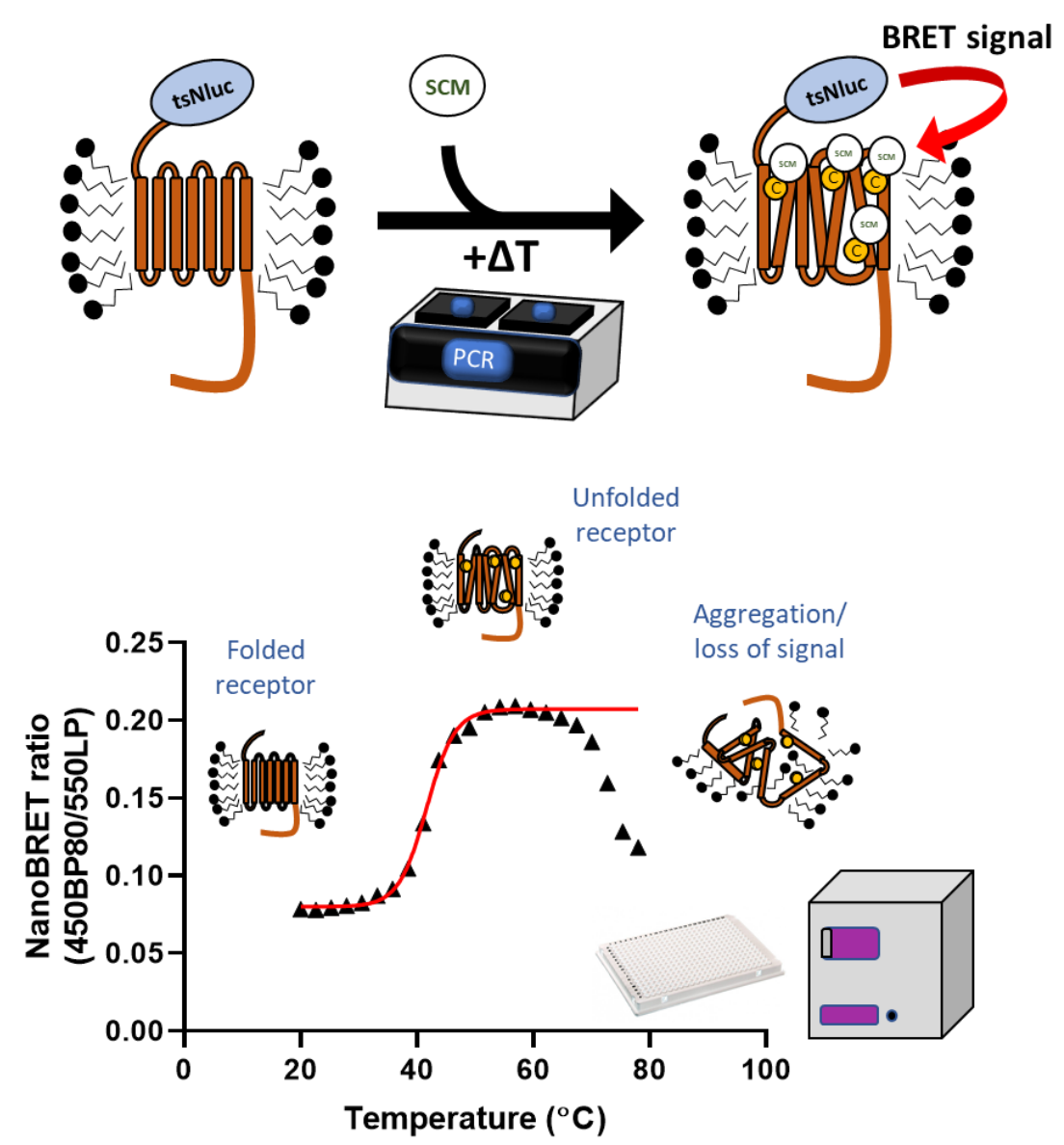

Figure 1: Principle of ThermoBRET assay measured in 384-well plate format. Detergent solubilised non-purified membrane preparations expressing GPCRs fused at the N-terminus with Nluc (or tsNluc) are heated using a PCR thermocycler in the presence of sulfo-Cy3 maleimide (SCM). As the protein unfolds due to thermal denaturation, SCM reacts with newly exposed cysteine residues putting the sulfo-Cy3 acceptor fluorophore in proximity with the Nluc donor. At higher temperatures, protein aggregation leads to a decrease in the NanoBRET signal and these points are truncated before fitting to a Boltzmann sigmoidal equation to obtain a melting point $\left(T_{m}\right)$. 
A
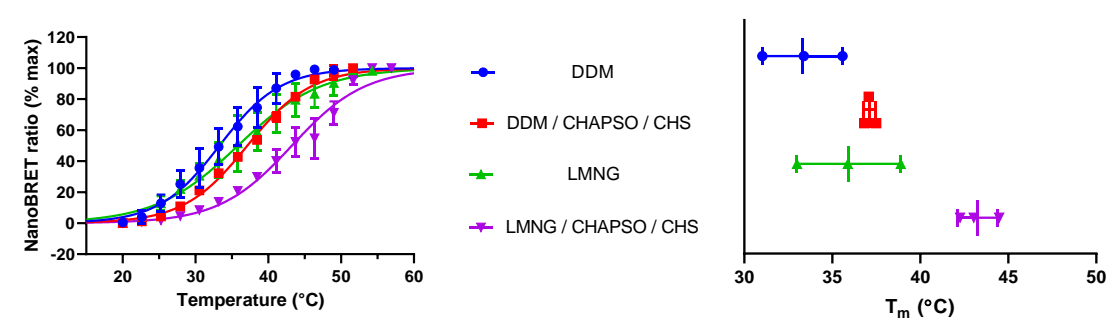

B

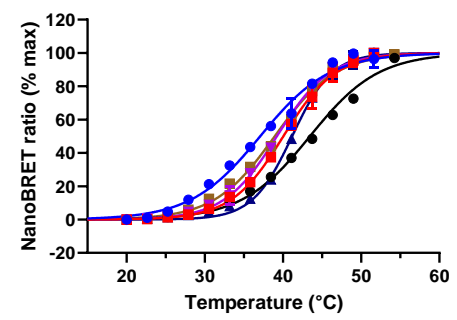

$$
\begin{aligned}
& \rightarrow \quad \text { No ligand } \\
& + \text { SR144528 } \\
& -\quad \text { AEA } \\
& \rightarrow \quad 2 A G \\
& \rightarrow \text { Cannabinol } \\
& -\quad \text { HU308 }
\end{aligned}
$$

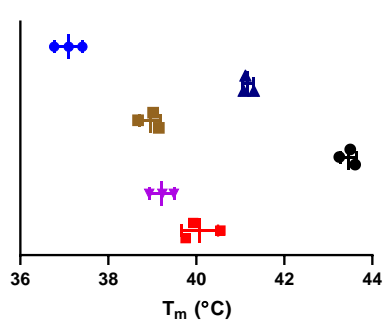

C

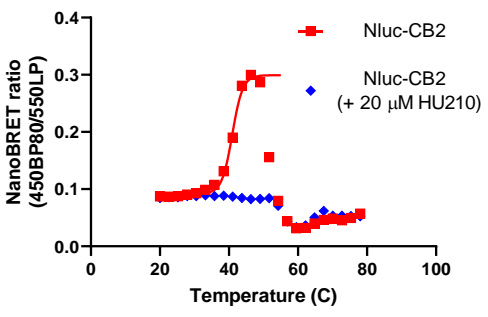

D

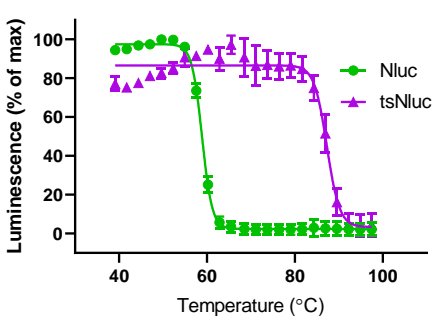

E

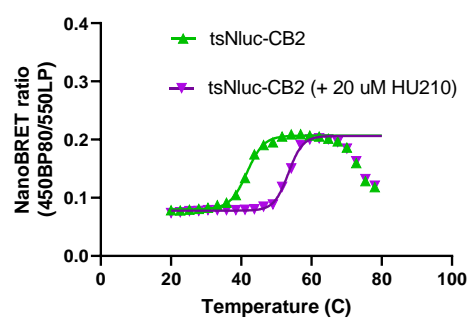

Figure 2: ThermoBRET measurements in different detergent conditions and with stabilising ligands, demonstrating superior performance of tsNluc over Nluc for high thermostability situations. ThermoBRET thermostability curves and pooled $T_{m}$ measurements for (A) Nluc- $\mathrm{CB}_{2}$ solubilised in the indicated detergent conditions. (B) in DDM/CHAPSO/CHS, in the presence/absence of ligands. (C) ThermoBRET curve for Nluc-CB2 solubilised in DDM / CHAPSO / CHS, showing that the curve for the receptor bound to HU210 cannot be fitted as it is stable beyond the point of Nluc stability. (D) Luminescence thermostability curves of purified Nluc and tsNluc. (E) ThermoBRET using tsNluc- $\mathrm{CB}_{2}$ in the presence/absence of HU210, showing a full fit for both curves. (A) and (B) show pooled normalised data showing mean \pm standard deviation for the number of experimental replicates evident in the far-right graph $(n \geq 2)$. (C) and (E) are raw fitted data from a single experiment performed 3 times. (D) is pooled normalised data from 3 -independent experiments. 


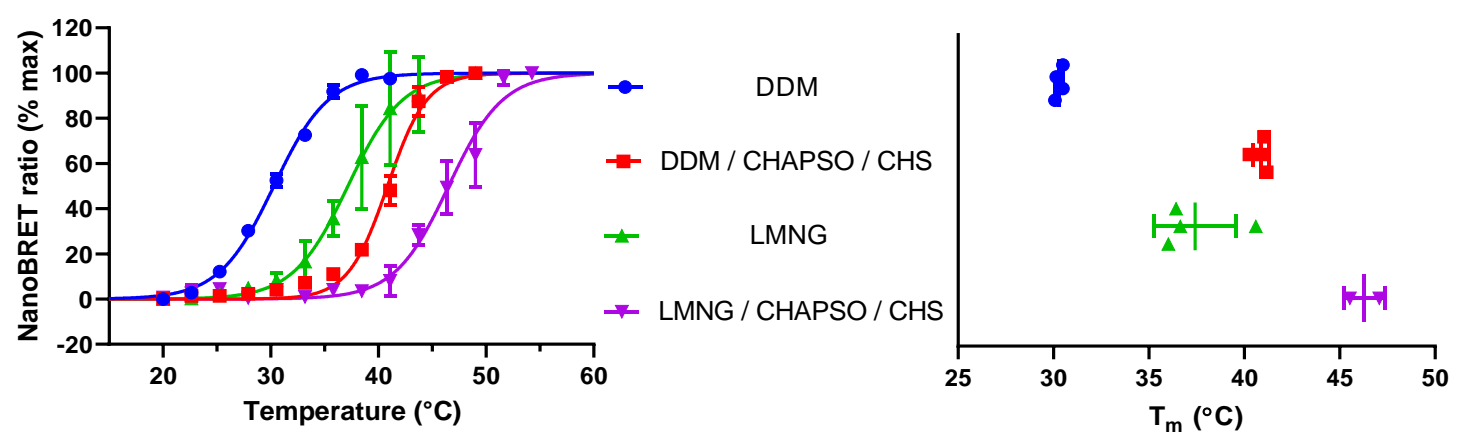

Supplementary Figure 1: Nluc- $\beta 2 A R$ thermostability in different detergent conditions. Data are pooled normalised values showing the mean \pm standard deviation for the number of experimental replicates evident in the far-right graph $(n \geq 2)$. 

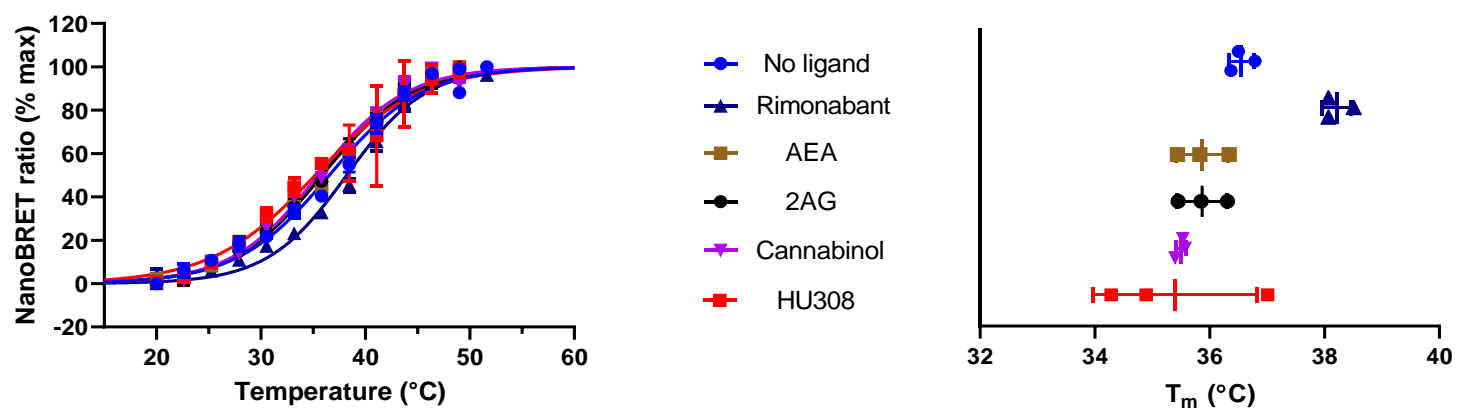

Supplementary Figure 2: Thermostability of Nluc-CB191-472 solubilised in DDM / CHAPSO / CHS. Data are pooled normalised values showing the mean \pm standard deviation for the number of experimental replicates evident in the far-right graph $(n \geq 3)$. All ligands were present at a concentration of $20 \mu \mathrm{M}$. 


\section{Supplementary Information 3: Amino acid alignment of Nluc and tsNluc}

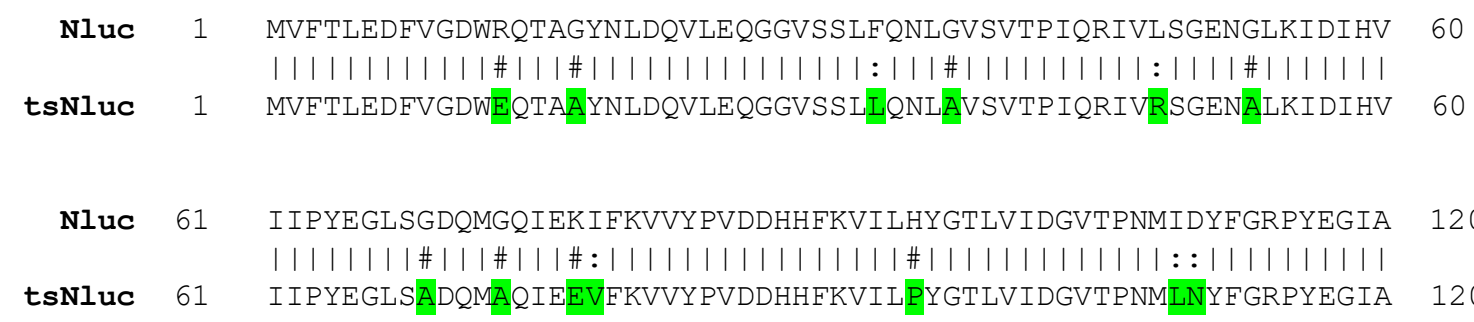

Nluc 121 VFDGKKITVTGTLWNGNKIIDERLINPDGSLLFRVTINGVTGWRLCERILA 171

Amino acid alignment of Nluc and tsNluc. The split Nanoluciferase fragment sequences of LgBit and HiBiT (VSGWRLFKKIS) were joined to create tsNluc. Residues shaded green show mutations of tsNluc from the Nanoluc sequence. Note the $\mathrm{C} 166 \mathrm{~F}$ mutation which results in tsNluc containing no cysteine residues. 


\section{Supplementary Information 4: Amino acid sequences of expression constructs used in this study.}

\section{> pcDNA4/TO SigPep-TwinStrep-Nluc-CB2}

MRLCIPQVLLALFLSMLTGPGEGSASDIGAPAFKSVQTGEFTAAAGSAWSHPQFEK GGGSGGGSGGSAWSHPQFEKGSGGSEDLMVFTLEDFVGDWRQTAGYNLDQVLE QGGVSSLFQNLGVSVTPIQRIVLSGENGLKIDIHVIIPYEGLSGDQMGQIEKIFKVVYP VDDHHFKVILHYGTLVIDGVTPNMIDYFGRPYEGIAVFDGKKITVTGTLWNGNKIIDE RLINPDGSLLFRVTINGVTGWRLCERILAPAGTMEECWVTEIANGSKDGLDSNPMK DYMILSGPQKTAVAVLCTLLGLLSALENVAVLYLILSSHQLRRKPSYLFIGSLAGADF LASVVFACSFVNFHVFHGVDSKAVFLLKIGSVTMTFTASVGSLLLTAIDRYLCLRYPP SYKALLTRGRALVTLGIMWVLSALVSYLPLMGWTCCPRPCSELFPLIPNDYLLSWLL FIAFLFSGIIYTYGHVLWKAHQHVASLSGHQDRQVPGMARMRLDVRLAKTLGLVLA VLLICWFPVLALMAHSLATTLSDQVKKAFAFCSMLCLINSMVNPVIYALRSGEIRSSA HHCLAHWKKCVRGLGSEAKEEAPRSSVTETEADGKITPWPDSRDLDLSDC*

\section{$>$ pcDNA4/TO SigPep-TwinStrep-Nluc-B2AR}

MRLCIPQVLLALFLSMLTGPGEGSASDIGAPAFKSVQTGEFTAAAGSAWSHPQFEK GGGSGGGSGGSAWSHPQFEKGSGGSEDLMVFTLEDFVGDWRQTAGYNLDQVLE QGGVSSLFQNLGVSVTPIQRIVLSGENGLKIDIHVIIPYEGLSGDQMGQIEKIFKVVYP VDDHHFKVILHYGTLVIDGVTPNMIDYFGRPYEGIAVFDGKKITVTGTLWNGNKIIDE RLINPDGSLLFRVTINGVTGWRLCERILAPAGTMGQPGNGSAFLLAPNGSHAPDHD VTQQRDEVWVVGMGIVMSLIVLAIVFGNVLVITAIAKFERLQTVTNYFITSLACADLV MGLAVVPFGAAHILMKMWTFGNFWCEFWTSIDVLCVTASIETLCVIAVDRYFAITSP FKYQSLLTKNKARVIILMVWIVSGLTSFLPIQMHWYRATHQEAINCYANETCCDFFT NQAYAIASSIVSFYVPLVIMVFVYSRVFQEAKRQLQKIDKSEGRFHVQNLSQVEQDG RTGHGLRRSSKFCLKEHKALKTLGIIMGTFTLCWLPFFIVNIVHVIQDNLIRKEVYILLN WIGYVNSGFNPLIYCRSPDFRIAFQELLCLRRSSLKAYGNGYSSNGNTGEQSGYHV EQEKENKLLCEDLPGTEDFVGHQGTVPSDNIDSQGRNCSTNDSLL*

\section{> pcDNA4/TO SigPep-TwinStrep-Nluc-CB1(91-472)\#}

MRLCIPQVLLALFLSMLTGPGEGSASDIGAPAFKSVQTGEFTAAAGSAWSHPQFEK GGGSGGGSGGSAWSHPQFEKGSGGSEDLMVFTLEDFVGDWRQTAGYNLDQVLE QGGVSSLFQNLGVSVTPIQRIVLSGENGLKIDIHVIIPYEGLSGDQMGQIEKIFKVVYP VDDHHFKVILHYGTLVIDGVTPNMIDYFGRPYEGIAVFDGKKITVTGTLWNGNKIIDE RLINPDGSLLFRVTINGVTGWRLCERILAPAGTENEENIQCGENFMDIECFMVLNPS QQLAIAVLSLTLGTFTVLENLLVLCVILHSRSLRCRPSYHFIGSLAVADLLGSVIFVYS FIDFHVFHRKDSRNVFLFKLGGVTASFTASVGSLFLTAIDRYISIHRPLAYKRIVTRPK AVVAFCLMWTIAIVIAVLPLLGWNCEKLQSVCSDIFPHIDETYLMFWIGVTSVLLLFIV YAYMYILWKAHSHAVRMIQRGTQKSIIIHTSEDGKVQVTRPDQARMDIRLAKTLVLIL VVLIICWGPLLAIMVYDVFGKMNKLIKTVFAFCSMLCLLNSTVNPIIYALRSKDLRHAF RSMFPSCEGTAQPLDNSMGDSDCLHKHANNAASVHRAAESCIKSTVKIAKVTMSV STDTSAEAL*

\#The full-length CB1 receptor contains an unusually long (around 117 amino acids) and likely unstructured $\mathrm{N}$-terminal domain. It was therefore truncated at the $\mathrm{N}$-terminus in order to bring the Nanoluc tag in proximity with the transmembrane helices. 


\section{> pcDNA4/TO SigPep-TwinStrep-tsNluc-CB2}

MRLCIPQVLLALFLSMLTGPGEGSASDIGAPAFKSVQTGEFTAAAGSAWSHPQFEK GGGSGGGSGGSAWSHPQFEKGSGGSEDLMVFTLEDFVGDWEQTAAYNLDQVLE QGGVSSLLQNLAVSVTPIQRIVRSGENALKIDIHVIIPYEGLSADQMAQIEEVFKVVYP VDDHHFKVILPYGTLVIDGVTPNMLNYFGRPYEGIAVFDGKKITVTGTLWNGNKIIDE RLITPDGSMLFRVTINGVSGWRLFKKISPAGTMEECWVTEIANGSKDGLDSNPMKD YMILSGPQKTAVAVLCTLLGLLSALENVAVLYLILSSHQLRRKPSYLFIGSLAGADFLA SVVFACSFVNFHVFHGVDSKAVFLLKIGSVTMTFTASVGSLLLTAIDRYLCLRYPPSY KALLTRGRALVTLGIMWVLSALVSYLPLMGWTCCPRPCSELFPLIPNDYLLSWLLFI AFLFSGIIYTYGHVLWKAHQHVASLSGHQDRQVPGMARMRLDVRLAKTLGLVLAVL LICWFPVLALMAHSLATTLSDQVKKAFAFCSMLCLINSMVNPVIYALRSGEIRSSAHH CLAHWKKCVRGLGSEAKEEAPRSSVTETEADGKITPWPDSRDLDLSDC*

\section{$>$ pJ411 His-TEV-Nluc}

MKKHHHHHHHHHHENLYFQGGSVFTLEDFVGDWRQTAGYNLDQVLEQGGVSSLF QNLGVSVTPIQRIVLSGENGLKIDIHVIIPYEGLSGDQMGQIEKIFKVVYPVDDHHFKV ILHYGTLVIDGVTPNMIDYFGRPYEGIAVFDGKKITVTGTLWNGNKIIDERLINPDGSL LFRVTINGVTGWRLCERILA*

\footnotetext{
> pJ411 HIS-TEV-TsNluc

MKKHHHHHHHHHHENLYFQGGSVFTLEDFVGDWEQTAAYNLDQVLEQGGVSSLL QNLAVSVTPIQRIVRSGENALKIDIHVIIPYEGLSADQMAQIEEVFKVVYPVDDHHFKV ILPYGTLVIDGVTPNMLNYFGRPYEGIAVFDGKKITVTGTLWNGNKIIDERLITPDGS MLFRVTINGVSGWRLFKKIS*
} 


\section{REFERENCES}

Beckner, R. L., K. Gawrisch and A. Yeliseev (2019). "Radioligand Thermostability Assessment of Agonist-Bound Human Type 2 Cannabinoid Receptor." Biophysical Journal 116(3): 193a.

Beckner, R. L., L. Zoubak, K. G. Hines, K. Gawrisch and A. A. Yeliseev (2020). "Probing thermostability of detergent-solubilized CB2 receptor by parallel $\mathrm{G}$ protein-activation and ligand-binding assays." Journal of Biological Chemistry 295(1): 181-190.

Congreve, M., C. de Graaf, N. A. Swain and C. G. Tate (2020). "Impact of GPCR structures on drug discovery." Cell 181(1): 81-91.

Dart, M. L., T. Machleidt, E. Jost, M. K. Schwinn, M. B. Robers, C. Shi, T. A. Kirkland, M. P. Killoran, J. M. Wilkinson, J. R. Hartnett, K. Zimmerman and K. V. Wood (2018). "Homogeneous Assay for Target Engagement Utilizing Bioluminescent Thermal Shift." ACS medicinal chemistry letters 9(6): 546-551.

Dixon, A. S., M. K. Schwinn, M. P. Hall, K. Zimmerman, P. Otto, T. H. Lubben, B. L. Butler, B. F. Binkowski, T. Machleidt and T. A. Kirkland (2016). "NanoLuc complementation reporter optimized for accurate measurement of protein interactions in cells." ACS chemical biology 11(2): 400-408.

Fang, Y. (2012). "Ligand-receptor interaction platforms and their applications for drug discovery." Expert opinion on drug discovery 7(10): 969-988.

Gibson, D. G., L. Young, R.-Y. Chuang, J. C. Venter, C. A. Hutchison and H. O. Smith (2009). "Enzymatic assembly of DNA molecules up to several hundred kilobases." Nature methods 6(5): 343-345.

Hall, M. P., J. Unch, B. F. Binkowski, M. P. Valley, B. L. Butler, M. G. Wood, P. Otto, K. Zimmerman, G. Vidugiris and T. Machleidt (2012). "Engineered luciferase reporter from a deep sea shrimp utilizing a novel imidazopyrazinone substrate." ACS chemical biology 7(11): 1848.

Hall, M. P., J. Unch, B. F. Binkowski, M. P. Valley, B. L. Butler, M. G. Wood, P. Otto, K. Zimmerman, G. Vidugiris, T. Machleidt, M. B. Robers, H. A. Benink, C. T. Eggers, M. R. Slater, P. L. Meisenheimer, D. H. Klaubert, F. Fan, L. P. Encell and K. V. Wood (2012). "Engineered luciferase reporter from a deep sea shrimp utilizing a novel imidazopyrazinone substrate." ACS Chem Biol 7(11): 1848-1857.

Hauser, A. S., M. M. Attwood, M. Rask-Andersen, H. B. Schioth and D. E. Gloriam (2017). "Trends in GPCR drug discovery: new agents, targets and indications." Nat Rev Drug Discov 16(12): 829-842.

Layton, C. J. and H. W. Hellinga (2010). "Thermodynamic Analysis of Ligand-Induced Changes in Protein Thermal Unfolding Applied to High-Throughput Determination of Ligand Affinities with Extrinsic Fluorescent Dyes." Biochemistry 49(51): 10831-10841.

Martinez, N. J., R. R. Asawa, M. G. Cyr, A. Zakharov, D. J. Urban, J. S. Roth, E. Wallgren, C. Klumpp-Thomas, N. P. Coussens and G. Rai (2018). "A widely-applicable high-throughput cellular thermal shift assay (CETSA) using split Nano Luciferase." Scientific reports 8(1): 116.

Milic, D. and D. B. Veprintsev (2015). "Large-scale production and protein engineering of G protein-coupled receptors for structural studies." Front Pharmacol 6: 66.

Pertwee, R. G. (2012). "Targeting the endocannabinoid system with cannabinoid receptor agonists: pharmacological strategies and therapeutic possibilities." Philosophical Transactions of the Royal Society B: Biological Sciences 367(1607): 3353-3363.

Tate, C. G. (2010). Practical considerations of membrane protein instability during purification and crystallisation. Heterologous Expression of Membrane Proteins, Springer: 187-203. 
Tippett, D., B. Hoare, T. Miljus, D. A. Sykes and D. Veprintsev (2020). "ThermoFRET: A novel nanoscale $G$ protein coupled receptor thermostability assay functional in crude solubilised membrane preparations." BioRxiv.

van Veen, H. W., K. Vukoti, T. Kimura, L. Macke, K. Gawrisch and A. Yeliseev (2012). "Stabilization of Functional Recombinant Cannabinoid Receptor CB2 in Detergent Micelles and Lipid Bilayers." PLoS ONE 7(10).

Vukoti, K., T. Kimura, L. Macke, K. Gawrisch and A. Yeliseev (2012). "Stabilization of functional recombinant cannabinoid receptor CB2 in detergent micelles and lipid bilayers." Plos one 7(10).

White, C. W., E. K. Johnstone, H. B. See and K. D. Pfleger (2019). "NanoBRET ligand binding at a GPCR under endogenous promotion facilitated by CRISPR/Cas9 genome editing." Cellular signalling 54: 27-34.

Zhang, X., R. C. Stevens and F. Xu (2015). "The importance of ligands for G protein-coupled receptor stability." Trends in biochemical sciences 40(2): 79-87. 